How to reference this article

Mazzarisi, P. (2020). Dati iniziali sulla reduplicazione linguistica nella lingua letteraria vigatese: la reduplicazione degli aggettivi tra diegesi e mimesi. Italica Wratislaviensia, 11(2), 17-39.

DOI: http://dx.doi.org/10.15804/IW.2020.11.2.2

\author{
Pietro Mazzarisi \\ Università degli Studi di Modena e Reggio Emilia, Italia \\ pietro.mazzarisi@unimore.it \\ ORCID: 0000-0002-2208-9398
}

\title{
DATI INIZIALI SULLA REDUPLICAZIONE LINGUISTICA NELLA LINGUA LETTERARIA VIGATESE: LA REDUPLICAZIONE DEGLI AGGETTIVI TRA DIEGESI E MIMESI
}

\section{PRELIMINARY DATA ON LINGUISTIC REDUPLICATION IN THE LITERARY LANGUAGE OF VIGATESE: THE ADJECTIVES' REDUPLICATION BETWEEN DIEGESIS AND MIMESIS}

\begin{abstract}
The article presents the results of research on linguistic reduplication in Vigatese, the literary language invented by writer Andrea Camilleri. This is an aspect of Camilleri's storytelling that is often noted by scholars but that has not yet received a dedicated study. The research is conducted on the corpus of 101 texts starring Salvo Montalbano - composed of 29 novels and 72 short stories published between 1994 and 2020 - within which about 300 different reduplication forms and more than 2,200 total occurrences have been recorded. Here, the data on the expressive reduplication with adjectives are given: 64 forms with 379 total occurrences analysed for different literary scales, at sentence level and paragraph level. The collocations within the corpus, the possible spelling variations, and whether the use is in mimesis or diegesis are restored. The methodological approach is of a mixed-methods nature: a digitally assisted text analysis (DATA) following the concept of scalable reading (Mueller, 2014) follows the traditional close-reading analysis. A second analysis of the data on the diegetic and mimetic axis was carried out. The results obtained were then used for a data-driven comparison conducted on the corpus of all of Giovanni Verga's narrative works.

These initial results show that three out of every four reduplications are a diegetic recurrence. Despite the extensive use of mimesis in the texts, the true orality of Camillerian storytelling seems to reside elsewhere. This research is part of a broader study of all narrative texts under the general hypothesis that the formal equivalents of the writer's popularity are to be found in the repetitiveness that was originally, and perhaps too hastily, criticised.
\end{abstract}

Keywords: linguistic reduplication, literature, Vigatese, Sicilian dialect, Camilleri 


\section{INTRODUZIONE}

\vigatese è una lingua letteraria, riconosciuta con l'autore che l'ha 1 inventata, Andrea Camilleri, e la Sicilia da cui hanno origine. Una lingua che, a Roma, si è misurata con l'italiano per farsi capire in tutta Italia. Difficile da tradurre, è tradotta in almeno 30 lingue $^{1}$.

In questo articolo presento i dati sull'impiego della reduplicazione espressiva degli aggettivi in vigatese. La ricerca, condotta sul corpus dei 101 testi pubblicati con protagonista il commissario Montalbano, ha registrato quasi 300 differenti forme reduplicative e più di 2.200 occorrenze. Il lavoro si distingue offrendo un ulteriore aspetto: ogni occorrenza del dataset specifica se l'uso è diegetico o mimetico, oltre alle varianti ortografiche. Le 64 forme reduplicative di aggettivi, o di altre parti del discorso in funzione aggettivale qui presentate, coprono il $22 \%$ di tutte le forme e il $17 \%$ delle occorrenze.

L'analisi dell'impiego delle reduplicazioni è parte di uno studio più ampio che prevede l'analisi delle cifre linguistiche di tutta la produzione del narratore. L'ipotesi di ricerca è che, nel caso della narrazione di Andrea Camilleri, i corrispettivi formali della popolarità editoriale si possano rintracciare ed esplicare tanto più con la ricerca del ripetuto che non con la più diffusa e tradizionale attività orientata soprattutto alla ricerca degli elementi rari. L'articolo dedica: il capitolo 2 alla ricezione e agli studi del vigatese; il capitolo 3 alla reduplicazione linguistica; il capitolo 4 al corpus principale della ricerca, alla metodologia impiegata e al corpus scelto per una comparazione data driven; il capitolo 5 alla presentazione e all'analisi dei risultati; il capitolo 6 a una correlazione che accompagna le conclusioni.

La parte finale dell'articolo mette in comparazione particolare e in correlazione generale i dati ottenuti. In particolare, nel cap. 5 il $d a-$ taset ottenuto dal corpus camilleriano è usato per una comparazione data driven con i 101 testi che compongono tutto il corpus narrativo di Giovanni Verga (10 romanzi e 91 novelle). In generale, nel cap. 6

1 L'elenco delle traduzioni dei libri di Camilleri è consultabile su www.vigata.org, unico sito vistato dall'autore. 
l'impiego della reduplicazione è messo in correlazione agli altri aspetti dello storytelling camilleriano che cooccorrono a creare quell'effetto di ripetitività criticato da quando il "caso Camilleri" si è imposto nel mercato editoriale italiano, ma qui ritenuto funzionale agli scopi narrativi autoriali.

\section{RICEZIONE E STUDI DEL VIGATESE}

Il vigatese appare letterariamente tra il 1978 e il 1980 in Il corso delle cose e in Un filo di fumo ${ }^{2}$ e fino alla morte dell'autore, nell'estate del 2019 a 93 anni, ne ha distinto la maggior parte della produzione narrativa. Questa comincia a imporsi sul mercato editoriale dalla metà degli anni Novanta, ma, a inizio del nuovo millennio, l'arrivo sotto la lente critica è controverso. I numeri delle copie vendute, la pubblicazione dei due Meridiani, i primi convegni e seminari in ambito isolano (sia Sicilia che Sardegna) testimoniano una significativa ricezione positiva, mentre diversi autorevoli studiosi, scrittori e intellettuali giungono a ritenere tale narrazione inefficace, esagerata, folcloristica. Lo scarto tra le ricezioni giustifica la dicitura di un "caso" Camilleri, altrimenti denominato solo "successo".

Simonetta Agnello Hornby, altra affermata scrittrice con numerose traduzioni e un forte legame con l'agrigentino, dà un sunto della ricezione. Emigrata a Londra dal 1972, apprezzata e premiata fin dall'esordio con il romanzo La mennulara nel 2002, ritiene che "il grande successo della serie di Montalbano, conzato di una generosa manciata di sarcasmo e un pizzico di supponenza da parte degli invidiosi, ha distolto l'attenzione del mondo accademico e della critica, ma non quella dei lettori, dalle altre opere che Camilleri regolarmente regala al suo pubblico: immaginative, sorprendenti, dotte, argute e dense di pensiero. Queste, assieme alla serie di Montalbano, rendono Camilleri il più grande scrittore

2 Il primo edito originariamente da Lalli nel 1978, il secondo da Garzanti nel 1980, sono stati riediti da Sellerio rispettivamente nel 1998 e nel 1997. 
siciliano del dopoguerra" (Agnello Hornby, 2016, p. 30. "Conzato", dal siciliano conza, in italiano "condito"3).

Tanti lavori trattano il vigatese, più o meno direttamente, poiché lo studio della produzione camilleriana difficilmente n'è scisso. Camilleri ne ha ampiamente discusso motivazioni e aspetti, tra gli altri, con Sorgi (2000), con Lodato (2002) e con de Mauro (2014). Dall'altra parte, mancava ad oggi una trattazione dedicata, sistematica e approfondita dell'abbondante impiego di forme reduplicative comunque già notato da più studiosi ${ }^{4}$.

Tra le monografie e le curatele, Demontis (2001) inquadra l'invenzione camilleriana entro la sperimentazione linguistica gaddiana. Il caso Camilleri. Letteratura e storia raccoglie gli atti del convegno tenutosi a Palermo 1'8-9 marzo 2002. Un'altra raccolta di atti, ma di un seminario a Cagliari, è Lingua, storia, gioco e moralità nel mondo di Andrea Camilleri curata da Marci (2004). Palumbo (2005) prende in analisi il progetto linguistico partendo dai risultati editoriali. Il glossario in calce a Un filo di fumo, compilato da Camilleri su richiesta del primo editore, Garzanti, è analizzato da Trainito (2008). Santulli (2010) prende in analisi gli esempi linguistici commentati dall'autore tramite il suo personaggio più famoso e che vanno dalla semantica alla sociolinguistica e alla pragmatica. Cerrato (2012) offre un'altra monografia con taglio sociolinguistico e dialettologico, ma estesa anche agli altri filoni narrativi dell'autore. Scaglia (2013) presenta uno studio in chiave estetica sul vigatese. Guastella (2015) propone uno strumento interpretativo delle scelte linguistiche operate dall'autore per il vigatese. Gran teatro $\mathrm{Ca}$ milleri, a cura di Nigro (2015), raccoglie studiosi italiani, fra gli altri, di italianistica, filologia, filosofia del linguaggio. Un'altra curatela con interessanti contributi di studiosi, questa volta di diverse nazioni europee, è quella di Curcio (2017). Oltre a queste monografie e curatele citate, si ricordano i due Meridiani dedicati all'autore e contenenti, insieme a una

3 I dizionari consultati sono Bonfiglio (2009), Piccitto (1977-2002), Mortillaro (1853), Biundi (1851).

${ }^{4}$ Come tratto distintivo della scrittura impiegato per marcarne la sintassi dialettale (Matt, 2020, p. 56) e, più generalmente, in associazione alla resa di uno stile improntato all'oralità (Valenti, 2014, pp. 241-242). 
selezione di romanzi preediti all'epoca, contributi di terzi sul vigatese: Andrea Camilleri. Storie di Montalbano 1994-2002 a cura di Mauro Novelli (2002) e Andrea Camilleri. Romanzi storici e civili a cura di Salvatore Silvano Nigro (2004). Fra le altre fonti si ricorda: la collana Quaderni camilleriani che dal 2016 raccoglie contributi critici; il progetto online CamillerINDEX, condotto da Diana, Marci e Ruggerini ${ }^{5}$, propone una raccolta di indici analitici sul vigatese e ha per obiettivo l'indicizzazione di tutta la produzione dell'autore; nondimeno, in diversi saggi critici apparsi in riviste si possono ricavare preziose informazioni sul vigatese, in particolare tra gli studi di natura traduttologica.

\section{REDUPLICAZIONE LINGUISTICA}

La reduplicazione linguistica viene definita come "the systematic repetition of phonological material within a word for semantic or grammatical purposes [...], a widely used morphological device in a substantial number of the languages spanning the globe" (Rubino, 2005a, p. 11) ${ }^{6}$, talmente largamente condivisa dalle lingue naturali che Edith A. Moravcsik ne ha proposto lo status di universale linguistico, all'interno della nota curatela di Greenberg (1978) su quegli aspetti del linguaggio accomunanti, per implicazione o esclusione, tutti gli idiomi. Questo status è stato approfondito in tempi più recenti da Stolz, Stroh e Urdze (2011), ma rimane ancora oggi oggetto di dibattito scientifico. La reduplicazione linguistica appare comunque, sempre secondo Carl Rubino ma all'interno del World Atlas of Language Structure, "a much more pervasive phenomenon than someone coming from a Western European world view might imagine" (2005b, p. 115) ${ }^{7}$.

In linguistica si identificano, a livello d'analisi formale, due tipi di reduplicazione: la reduplicazione totale (complete, full o total redupli-

\footnotetext{
5 https://www.camillerindex.it

6 "la ripetizione sistematica di materiale fonologico all'interno di una parola con scopi semantici o grammaticali [...], un procedimento morfologico largamente impiegato da un considerevole numero di lingue sparse in tutto il mondo".

7 "un fenomeno molto più pervasivo di quanto si possa immaginare venendo da una visione del mondo dell'Occidente europeo".
} 
cation) e la reduplicazione parziale (partial reduplication). La prima "is the repetition of any morphological unit, preferably from the root up to the whole word", la seconda "can manifest itself in any way from simply reduplicating a consonant and vowel to the almost complete repetition of a base" (Schwaiger, 2015, pp. 468-469) ${ }^{8}$. La reduplicazione espressiva di aggettivi viene considerata una forma occasionale di intensificazione semantica, come in grande grande. L'aggettivo reduplicato può anche cristallizzarsi in locuzione come nell'italiano quatto quatto e cambiare parte del discorso come in papale papale e in così così: "un film così così". Per approfondimenti sulla reduplicazione linguistica in italiano si rimanda a Wierzbicka (1986) e Thorton (2008; 2009).

\section{CORPUS E METODOLOGIA}

Il corpus è composto da 29 romanzi e 72 racconti. Si riporta l'anno della prima pubblicazione dei romanzi e l'anno della prima pubblicazione in volume dei racconti. Alcuni romanzi sono stati successivamente riediti presso altro editore, come visto al cap. 2 per i Meridiani della Mondadori, alcuni racconti erano stati precedentemente editi in giornali o riviste. Nelle fonti bibliografiche primarie è indicato l'anno di prima pubblicazione, il titolo, l'edizione/ristampa consultata tra parentesi e la lista delle abbreviazioni ${ }^{9}$.

I romanzi, tra i quali Acqua in bocca è l'unico scritto insieme a un altro autore, Carlo Lucarelli, sono stati pubblicati tra il 1994 e il 2020. Eccetto il testo scritto insieme a Lucarelli ed edito per i tipi di Minimum Fax, tutti i romanzi sono stati pubblicati da Sellerio. Tra i racconti,

8 “[La reduplicazione totale] è la ripetizione una qualsiasi unità morfologica, preferibilmente dalla radice fino all'intera parola", "[la reduplicazione parziale] può apparire in una qualsiasi forma dalla semplice reduplicazione di una consonante e una vocale fino alla quasi completa ripetizione della base".

9 L'ultimo romanzo del corpus, Riccardino, è stato scritto nel 2005, aggiornato nella sola veste linguistica nel 2016, quindi edito postumo nel 2020 sia in edizione singola che in edizione accompagnata dalla prima stesura. Vengono conteggiate le sole reduplicazioni presenti nella stesura definitiva del 2016 abbreviata in RI, mentre in nota vengono segnalate le eventuali differenze rispetto alla prima stesura del 2005 abbreviata in R. 
4 sono stati pubblicati in volumi che comprendono anche racconti di altri scrittori, i restanti 68 in volumi che raccolgono solo racconti dell'autore empedoclino. Questi 72 racconti sono stati pubblicati tra il 1999 e il 2018 in questo ordine: 30 racconti nella raccolta Un mese con Montalbano (1999) edita da Mondadori; 20 racconti nella raccolta Gli arancini di Montalbano (1999) edita da Mondadori; 6 racconti nella raccolta La paura di Montalbano (2002) edita da Mondadori; 3 racconti nella raccolta La prima indagine di Montalbano (2005) edita da Mondadori; il racconto La finestra sul cortile, quale unico inedito di una raccolta di racconti precedentemente pubblicati, in Racconti di Montalbano (2008) edita da Mondadori; il racconto Una cena speciale in una raccolta di racconti di autori misti Capodanno in giallo (2012) edita da Sellerio; il racconto Notte di Ferragosto in una raccolta di racconti di autori misti Ferragosto in giallo (2013) edita da Sellerio; 8 racconti nella raccolta Morte in mare aperto e altre indagini del giovane Montalbano (2014) edita da Sellerio; il racconto La calza della Befana in una raccolta di racconti di autori misti Un anno in giallo (2017) edita da Sellerio; il racconto Ventiquattr'ore di ritardo in una raccolta di racconti di autori misti Una giornata in giallo (2018) edita da Sellerio.

L'approccio metodologico impiegato è di natura mixed methods. A una analisi classica dei testi con annotazione manuale delle occorrenze e delle diverse varianti, diglossiche, grammaticali e ortografiche, in cui la stessa forma reduplicativa può comparire, è seguita una digital assisted text analysis (DATA) di correzione dei risultati (Mueller, 2014) sulla copia digitale del testo. I dati così ottenuti sono stati oggetto di una seconda analisi sull'asse diegetico/mimetico. Con text mining i passi diegetici e gli scambi mimetici sono stati separati digitalmente al fine di evidenziare, per ogni singola occorrenza, la distinzione d'uso da parte del narratore e dei personaggi. Infine, con una ricerca comparativa data driven sono state ricercate le stesse semantiche reduplicative ${ }^{10}$ nel corpus narrativo verghiano composto da 10 romanzi e 91 novelle $^{11}$.

10 Ovvero se nel corpus camilleriano è registrato chino chino per pieno pieno, entrambe le reduplicazioni (e le relative forme flesse) sono state ricercate nel corpus verghiano.

11 I romanzi sono: I Malavoglia; Mastro-Don Gesualdo; Sulle lagune; Una peccatrice; Storia di una capinera; Eva; Tigre reale; Eros; Il marito di Elena; Dal tuo al 


\section{RISULTATI}

Le reduplicazioni impiegate dall'autore vengono presentate in ordine alfabetico rispettando le seguenti convenzioni ortografiche per comodità di chi legge: la reduplicazione è in corsivo e in neretto, seguita dalla traduzione in italiano tra virgolette alte se necessaria; l'abbreviazione in maiuscolo si riferisce a un romanzo, l'abbreviazione in maiuscolo corsivo si riferisce a una raccolta di racconti, l'abbreviazione in minuscolo si riferisce a un racconto; l'abbreviazione del romanzo o della raccolta di racconti precede il numero di pagina in cui la forma è stata registrata, vengono indicate tra parentesi le eventuali varianti della stessa forma reduplicativa se presenti e segue l'abbreviazione del titolo del racconto, laddove si tratta di un racconto presente in una raccolta del solo Camilleri; 1'abbreviazione del racconto precede il numero di pagina per i racconti contenuti in raccolte di autori vari e per l'unico inedito in Racconti di Montalbano, La finestra sul cortile.

Il numero di pagina marcato in corsivo indica che la forma è impiegata diegeticamente dal narratore; altrimenti, il numero di pagina non marcato indica che la forma reduplicativa è impiegata mimeticamente nel discorso diretto o all'interno di epistole. Un impiego che si discosti da quest'asse viene marcato in neretto e con sottolineatura.

Sono escluse dalla presentazione le tautologie, le ripetizioni a contatto, le reduplicazioni onomatopeiche non lessicalizzate ma episodiche (comunque registrate) e le diverse forme di reduplicazioni e ripetizioni circoscritte nell'uso al solo personaggio Catarella.

\section{Reduplicazioni espressive: aggettivi}

'Nnuccenti 'nnuccenti, "innocente innocente": MM 284 (y).

(quello che) c'è scritto c'è scritto, "proprio quella cosa è scritta": VV 95.

Assà assà, "assai assai": cs 30; COV 137; vor 28.

mio. Per l'elenco delle 91 novelle cf.: https://www.liberliber.it/mediateca/libri/v/verga/ tutte_le_novelle/html/index.htm 
Avaro avaro: ON 170.

Bianca bianca: PM 227 (pm); nf 29; ACF 25; RP 98. ${ }^{12}$

Bidienti bidienti, "ubbidiente ubbidiente": PF 89.

Bono bono, "buono buono": LL 110; nf 34.

Brevi brevi: vor 28 .

Bruna bruna: RP 98.

Calmo calmo: MM 213 (v); AM 252 (mav); VA 242; MMA 310 (carmo carmo, lo); CA 18 (carmo carmo). ${ }^{13}$

Cattivello cattivello: GT 137.

Càvudo càvudo, "caldo caldo": LM 117 (càvude càvude); GT 264 (càvuda càvuda); PR 143 (cavudo cavudo); PS 113; CAV 204 (càvudi càvudi); GIOCS 80 (caldi caldi); MMA 297 (càvuda càvuda, lo); RP 174 (càvudi càvudi).

Chiaro chiaro: VV 152 (chiari chiari), 153 (chiari chiari); MM 219 (chiara chiara, v), 286 (chiari chiari, y); AM 163 (sep), 249 (chiara chiara, mav); ON 111 (chiara chiara); GB 80 (chiari chiari); SA 12 (chiara chiara); VN 170.

Chino chino, "pieno pieno":AS 218 (china china); PS 87, 87, 208 (china china); CAV 157; DG 49 (china china), 83 (china china), 93 (china china); CACT 10, 30, 45; SA 245; MMA 269 (al); ACF 30 (china china), 114, 144, 182; RP 18 (china china), 212 (china china); MC 36, 56 (chine chine), 87 (chine chine), 91, 129; vor 13 (china china); CA 12 (chini chini); RI 180 (china china).

Cilestri cilestri, "celestri celestri”: RP 98.

12 Alla reduplicazione con significante italiano bianca bianca sono accostate altre due forme reduplicative sempre in uso diegetico, giarna giarna con significante siciliano e pallita pallita con significante italiano sicilianizzato: tutte le relative occorrenze, con la sola eccezione di PR 217, sono riferite a figure femminili. Non è infatti insolito trovare tra gli usi due, o più, forme reduplicative diverse per uno stesso significato. Né lo è trovarle in un medesimo testo, come nel racconto nf dove si descrive l'impallidire della sorella della vittima con bianca bianca (nf 29) e della fidanzata della vittima con pallita pallita (nf 38 , cf. infra).

13 Le occorrenze e le forme ortografiche di calmo calmo danno una minima dimostrazione dell'evoluzione del vigatese. Tutte impiegate diegeticamente, variano dall'iniziale significante italiano calmo verso il significante dialettalizzato carmo, a un solo passo di distanza dal pieno significante siciliano oggi in uso, carmu. 
Comunista comunista: VV 67.

Così così, "mediocre": SA 98.

Cupo cupo: PM 102 (cpp).

Curto curto, "corto corto": GIOCS 24.

Duci duci, "dolce dolce": CACT 130.

Faccia a faccia, "tipologia di incontro oppositivo tra persone, idee, opinioni differenti": AM 196 (r).

Facile facile: CANT 112 (facili facili); MM 352 (c); AM 161 (sep); CAV

41 (facili facili). ${ }^{14}$

Fina fina, "fine fine": PS 209.

Fitto fitto: $A M 146$ (af); VA 184; DG 37; ACF 252 (fitta fitta).

Fresco fresco: CANT 149; MM 27 (ad); AM 127 (rp); GT 220; PR 232 (frisco frisco); PIM 183 (frisca frisca, pim); SA 20 (frisca frisca); RP 181 (frischi frischi); cb 22 (frischi frischi); CA 160 (frischi frischi).

Friddo friddo, "freddo freddo": VV 131; PM 167 (qs); VA 57 (fridda fridda).

Fritto e rifritto, "più volte cotto/ripetuto": COV 227; RI 247. ${ }^{15}$

Giarno giarno, "pallido pallido": CANT 224; LM 120 (giarna giarna); VV 178; ON 45 (giarna giarna), 206; PR 217 (giarna giarna); DG 166.

Granni granni, "grande grande": GIOSS 214; MC 175, 193.

Grosse grosse: GT 174.

14 Il dialetto siciliano non presenta distinzione formale tra il singolare e il plurale degli aggettivi qualificativi del secondo gruppo a causa di innalzamento vocalico dovuto a metafonia. La metafonia interessa le vocali atone, innalzando "e" in " $i$ " e "o" in "u". Pertanto, si impiega facili sia per sostantivi singolari che plurali.

15 In RI viene usata dall'autore all'interno del fax che occupa tutto il penultimo capitolo dell'epilogo narrativo delle indagini del commissario Montalbano. Si riferisce al consunto teatrino del rapporto mafia e politica che il personaggio Montalbano, volendo decidere in prima persona la propria vita, si ostina a perseguire nell'ultima indagine, mentre proprio con il fax l'autore Camilleri ne auspica un finale diverso. È l'ultimo confronto tra i due con pareri diversi sulla narrazione, ma non il primo. Il primo confronto avviene nel 1999 nel racconto Montalbano si rifiuta contenuto ne Gli arancini di Montalbano. 
Leggero leggero: CANT 16 (leggia leggia), 171 (leggera leggera); GT 144 (lèggio lèggio); ON 49 (leggio leggio); PM 14 (liggero liggero, gf); PR 58 (liggero liggero); PIM 117 (lèggio lèggio, pim); LC 99 (leggia leggia), 265 (liggera liggera); VA 35 (liggera liggera), 235 (liggero liggero), 263 (leggia leggia); AS 199 (liggero liggero); PS 9 (leggia leggia), 82 (leggio leggio); ED 254 (liggera liggera); DG 180 (liggero liggero); SA 100 (liggero liggero); GIOCS 24 (liggero liggero); COV 228 (liggero liggero); MC 16 (leggia leggia), 215 (leggia leggia), 282 (leggia leggia); RI 72 (liggero liggero).

Lenti lenti: PIM 180 (pim).

Lisci lisci: PIM 261 (ro).

Longo longo, "lungo lungo, alto alto": GT 178; VA 20 (longhe longhe); PS 87 (longhe longhe), 88; ACF 29; MC 56, 100, 170 (longhi longhi); RI 215 (longhe longhe). ${ }^{16}$

Luntana luntana, "lontana lontana": LC 195; VA 39 (luntanu luntanu); GIOSS 72.

Mala mala, "brutta brutta": ACF 171.

Minuto minuto, "con caratteri molto piccoli": GT 23.

Nico nico, "piccolo piccolo": FA 98 (nica nica); CANT 139 (nichi nichi); AM 87 (nica nica, crc); GT 231; ON 132 (nichi nichi); GB 60 (niche niche); PR 200 (nica nica); LC 134 (nica nica), 265 (nica nica); VA 19 (nichi nichi), 171 (nica nica); AS 93 (niche niche), 153 (niche niche), 188 (nica nica); PS 229 (nica nica); CAV 21, 113, 262 (nichi nichi); ED 102 (nica nica); DG 147, 208 (nica nica), 224; CACT 157 (nica nica), 195 (nica nica); GIOCS 121 (nica nica); VN 43 (nica nica); MMA 275 (al); ACF 167 (nichi nichi), 193 (niche niche); cb 36 (niche niche); MC 52 (nicareddro nicareddro), 91, 135 (nica nica), 175 (niche niche), 281 (nica nica); vor 14 (nica nica), 26 (niche niche), 31 (nica nica); RI 8 (nichi nichi), 116 (nica nica), 127 (nica nica), 127, 127, 191 (nica nica), 266. ${ }^{17}$

16 La forma è usata qui con i significati "lungo" o "alto" deducibili dal contesto. Come preposizione altrove: "Si susì dalla tavola che longo longo la schina gli serpeggiavano addrizzuna di friddu" (CAV 200).

17 L'occorrenza RI 8, riferita ai "tilefonini nichi nichi”, è diversa rispetto a $\mathrm{R} 8$, "telecamere niche niche". 
Nivuri nivuri, "neri neri”: PIM 183 (pim); LC 169 (nìvura nìvura); RI 131 (nìvuri nìvuri).

Novo novo, "nuovo nuovo": CANT 23 (nuovi nuovi); MM 16 (nova nova, la), 203 (nova nova, vl), 271 (nova nova, fd), 358 (novi novi, sc); GT 48 (nova nova), 173, 281 (novi novi); ON 11, 130 (nova nova), 131; GB 181 (nova nova); PIM 72 (nova nova, sl), 152 (nova nova, pim); LC 222 (nova nova); PS 85 (nova nova), 152; CAV 269 (nova nova); ED 135; CACT 174, 188; SA 48 (novi novi), 49 (nova nova), 55, 62 (novi novi); ACF 244 (nova nova); vor 11 (novi novi); CA 157 (nova nova); RI 52 (nova nova), 85.

Pallita pallita, "pallida pallida": DG 39; nf 38.

Pàmpini pàmpini, "(gli occhi diventano/si fanno) socchiusi, annebbiati, tremolanti”: CANT $187 .{ }^{18}$

Paro paro, "preciso, uguale, uniforme": FA 33 (para para); CANT 23

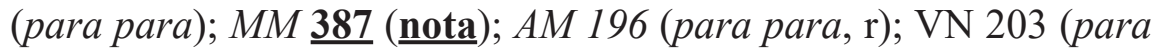
para). ${ }^{19}$

Preciso preciso, "identico, esatto, meticoloso": LM 130 (pricisi pricisi); MM 226 (precisa precisa, gl); AB 58.

18 Questa reduplicazione compare solo in CANT, insieme all'altra reduplicazione con lo stesso significato pupi pupi, in seguito opzione unica in tutto il corpus: "gli occhi gli facevano pupi pupi" (CANT 175); "gli occhi principiavano a fargli pàmpini pàmpini” (CANT 187). Non sono registrate nei dizionari. Sono sempre impiegate in riferimento agli occhi e con il verbo fare, tranne in "appena che la vista comenzava a fargli pupi pupi chiuiva il libro" (DG 9) e in "la tilevisioni le fa veniri i pupipupi nell'occhi” (ACF 247). Nel 1999 il gruppo musicale agrigentino Tinturia incide la canzone Occhi a pampina. La pampina è la foglia e l'espressione presa dal gergo giovanile indica gli occhi persi e serrati per uso di stupefacenti. Il sito vigata.org riferisce le reduplicazioni a quando gli occhi si socchiudono e le palpebre sbattono per stanchezza o sonno e all'annebbiarsi e lo sfocarsi della vista. Le traduzioni di CANT in inglese, francese, spagnolo e russo per pupi pupi sono: "his eyes clouding over", "ses yeux clignotaient", "se le nublaban los ojos”, “в глазах у него запрыгало"; per pàmpini pàmpini: "his eyes would start to droop", "ses yeux commençaient à clignoter", "se le empezaban a cerrar los ojos", “глаза у него принимались мигать”. Per riportare la costruzione tipica vigatese e parte del significato gergale si propone la categorizzazione aggettivale con i verbi diventare o farsi.

19 Un altro esempio qui di due forme reduplicative per uno stesso significato: paro paro e preciso preciso. 
Puliti puliti: MM 287 (dft); AM 66 (pulita pulita, co), 67 (pulita pulita, co); PR $253 .{ }^{20}$

Pupi pupi, "(gli occhi diventano/si fanno) socchiusi, annebbiati, tremolanti": CANT 175; LM 75; MM 247 (toca); PM 10 (gf), 146 (qs), 208 (qs); GB 133, 170; PIM 213 (pim); LC 159; CAV 103; DG 9, 139; SA 242; LL 77; ACF 247 (pupipupi).

Ricci ricci: VV 162 (riccio riccio); PM 27 (fm); RP 98; MC 37; CA 47, 196, 245.

Sano sano: AM 266 (sane sane, am); PM 266 (pm); PIM 9 (sanu sanu, sl); PS 77 (sana sana), 160 (sana sana), 198 (sana sana); CAV 23; ED 125; MC 46 (sana sana).

Scuro scuro: AM 128 (rp); GT 142-143; LC 27 (scure scure); RP 15; cb 36 (scuri scuri).

Semplice semplice: MM 67 (a), 89 (d43); AM 27 (semprici semprici, pmc); GT 51 (semplici semplici); ON 29 (semprici semprici); PS 242 (semplici semplici); DG 51 (semplici semplici).

Serio serio: LM 245; VV 206 (seria seria); MM 45 (pc); GT 251 (seria seria); ON 63, 85 (seria seria), 188; PM 242 (ms); PR 11; ED 91 (seria seria), 258 (seria seria); CACT 41; VN 187 (seria seria); PF 116, 181 (seria seria); ACF 15 (seria seria); RP 156; MC 167 (seria seria).

Sicco sicco, "secco secco": LM 18, 18; VV 46 (sicca sicca); MM 373 (mdt); AM 117 (rp); PM 27 (sicca sicca, fm), 127 (qs), 289 (ms); GB 62, 84, 119, 125; PIM 36 (sicca sicca, sl), 186 (pim), 196 (sicca sicca, pim); PS 88; ED 32, 138; CACT 171 (sicca sicca); SA 96; VN 33; PF 223; MMA 90 (mma), 201 (cmp); GIOSS 214; RP 36, 159; MC 74, 211; RI 117.

Sicuro sicuro: $A M 103$ (psai).

Sottili sottili: LC 116; PF 159; MC 175.

Strano strano: DG 203.

Stritto stritto, "stretto stretto": AM 190 (cfa); ON 60 (stritti stritti); PM 190 (qs); GB 25, 223; PR 28 (stritta stritta), 209 (stritta stritta), 211; PIM 229 (stritte stritte, pim); DG 21 (stritta stritta), 196 (stritti strit-

${ }^{20}$ In MM 287 (dft) l'impiego è dialettale con significato di "eleganti, ben vestiti". 
ti); SA 110 (stritti stritti), 130; GIOCS 31 (stritta stritta); COV 44 (stritti stritti); PF 195; ACF 36 (stritta stritta); RP 129; cb 11 (stritta stritta); CA 231 (stritti stritti); RI 11 (stritti stritti), 61 (stritte stritte), 215 (stritte stritte), 265 (stritti stritti). ${ }^{21}$

Tanto tanto: CAV 238; SA 39.

Tenneri tenneri, "teneri teneri": cs 43.

Terra terra, "grezzo, concreto, limitato": CANT 166; CACT 97.

Tisi tisi, "molto tesi": RI 28.

Tunna tunna, "rotonda rotonda": CAV 168.

Tutte tutte: MC 79, 79.

Vascio vascio, "basso basso": GT 46 (vascia vascia); LC 225 (vascie vascie); SA 165, 167 (vascie vascie).

Vivi vivi: AM 149 (af), 269 (am); PIM 180 (pim); DG 101 (vivo vivo).

Delle 64 forme reduplicative presentate, nel corpus verghiano ne sono state rintracciate solo 16 per 41 occorrenze totali, mai con significanti italiani sicilianizzati, tanto meno con significanti siciliani (le uniche variazioni sono nelle forme flesse, qui non riportate): bianco bianco, 2 occorrenze (Jeli il pastore; Fra le scene di vita); buoni buoni, 1 occorrenza (I Malavoglia, cap. 15); caldo caldo, 2 occorrenze (Tentazione!; Gli orfani); corto corto, 1 occorrenza (Eros, cap. 48); fresche fresche, 1 occorrenza (Mastro-Don Gesualdo, pt. 3, cap. 2); freddo freddo, 5 occorrenze (2 in Jeli il pastore; 2 ne I Malavoglia, cap. 4; 1 ne Il segno d'amore); grosso grosso, 2 occorrenze (...e chi vive si dà pace; MastroDon Gesualdo, pt. 4, cap. 5); leggero leggero, 2 occorrenze (Storia di una capinera, Catania 9 gennaio; Mastro-Don Gesualdo, pt. 1, cap. 7); lento lento, 2 occorrenze (Jeli il pastore; Eva); lungo lungo, 3 occorrenze (Il marito di Elena, cap. 2; Mastro-Don Gesualdo, pt. 4, cap. 5; Storia di una capinera, 10 ottobre); lontano lontano, 7 ricorrenze (2 ne I Malavoglia, capp. 10 e 11; 2 in Vagabondaggio; 1 in Storia di una capinera, monte Ilice 3 settembre 1854; 1 in Di là del mare; 1 in Quelli del colèra); minuta minuta, 1 occorrenza (Jeli il pastore); pallido pallido, 5 occorrenze (2 in Storia di una capinera, 24 giugno e Lunedì 7 aprile;

21 L'occorrenza RI 215 è assente in R. 
1 in Mastro-Don Gesualdo, pt. 1, cap. 4; 1 in Cos'è il re; 1 in Jeli il pastore); scuro scuro, 1 occorrenza (I Malavoglia, cap. 15); stretto stretto, 5 occorrenze (2 in Mastro-Don Gesualdo, pt. 4, cap. 5; 1 in Storia di una capinera, 20 novembre; 1 in Primavera; 1 in Giuramenti di marinaio); tutto tutto, 1 occorrenza (Né mai, né sempre!).

La circoscritta comparazione data driven rafforza l'indiziale maggiore ricorso dell'autore empedoclino alle forme reduplicate, infatti le stesse 16 forme in Camilleri registrano 98 occorrenze. È un dato iniziale che richiederà ulteriori analisi tramite comparazioni più estese. In prospettiva comparatistica, tra i testi e gli autori che sembrano maggiormente avvicinarsi agli usi camilleriani si distingue l'autobiografia Terra matta di Vincenzo Rabito. Le similarità con la narrazione di Rabito segnata da spontaneità linguistica vanno a supporto del lavorio linguistico di Camilleri nella direzione del cuntastorie per il proprio storytelling e saranno approfondite in un lavoro futuro.

\section{CONCLUSIONI}

Un dato che appare significativo viene evinto sull'asse diegesi/mimesi, analisi che sembra, in generale, promettente per lo studio della produzione camilleriana: le reduplicazioni espressive presentate sono usate 3 volte su 4 diegeticamente ${ }^{22}$. Il rapporto risulta il più alto rispetto alla categoria grammaticale. Seguono le reduplicazioni dei sostantivi con funzione aggettivale o prepositiva proprie del solo dialetto, $72 \%$ in diegesi e $28 \%$ in mimesi ${ }^{23}$. Le reduplicazioni espressive dei sostantivi, $65 \%$ in diegesi e $35 \%$ in mimesi ${ }^{24}$. Gli avverbi, i quali compongono la categoria maggiormente usata, mostrano $64 \%$ in diegesi e $35,5 \%$ in mime$\mathrm{si}^{25}$. I verbi riportano il rapporto più equilibrato, $48 \%$ in diegesi e $51 \%$

22 Le 64 forme coprono il $22 \%$ di tutte le forme e le 379 occorrenze coprono il 17\% di tutte le occorrenze. Di esse appunto 286, il 75,5\% sono usate diegeticamente e 91, il $24 \%$, mimeticamente.

23 Coprono l' $11 \%$ di tutte le forme e il 10\% di tutte le occorrenze.

24 Coprono il $16 \%$ di tutte le forme e il $7 \%$ di tutte le occorrenze.

25 Coprono il $27 \%$ di tutte le forme e il $57 \%$ di tutte le occorrenze. 
in mimesi ${ }^{26}$. Chiudono il conteggio le parole straniere, i pronomi, le interiezioni e la preposizione ${ }^{27}$, il cui basso numero di forme e occorrenze generali incide limitatamente sul dato complessivo del $65,4 \%$ in diegesi e $34,2 \%$ in mimesi di tutte le occorrenze entro il corpus.

Due volte su tre sembra un rapporto consistente di una oralità non tanto denotata dalla larga presenza di dialoghi, quanto nello stile ripetitivo. La natura fortemente orale della reduplicazione porterebbe a ipotizzarne, in ambito narrativo, un prevalente impiego nei dialoghi. L'analisi svolta in questa sede confuta una simile ipotesi: non sembra tanto Camilleri a usare la lingua dei personaggi, quanto i personaggi a usare la lingua di Camilleri. Si coglie un primo indizio dell'annullamento linguistico di ogni ipotetica distanza tra i tre vertici del triangolo autore, personaggi, narratore già nella nota prefatoria all'esordio narrativo Il corso delle cose: "l'autore [...] avendo immaginato una storia di fantasia, non ha saputo fare altro che calarla para para nelle case e nelle strade che conosce" (Camilleri, 1998, p. 11). Nella nota conclusiva alla raccolta di racconti Un mese con Montalbano l'autore Camilleri ne dà ulteriore conferma: "I racconti qui raccolti sono trenta. A leggerne uno al giorno ci si impiega un mese paro paro: questo vuole significare il titolo" (MM $\underline{\mathbf{3 8 7}})$. L'esclusione della figura del narratore diviene massima e definitiva nell'epilogo narrativo Riccardino.

In prospettiva correlativa, infine, per lo storytelling camilleriano si può avanzare la definizione di "ridondanza narrativa scalare": un reiterare continuo a più livelli di scale letterarie, di cui la reduplicazione è, a livello linguistico, una sola componente accanto alla ripetizione a contatto, ripetizione di clausole, modi di dire, proverbi, tic linguistici. Relativamente alle reduplicazioni espressive di aggettivi uno dei passaggi più rappresentativi è quando Ingrid, l'amica svedese di Montalbano conosciuta e aiutata nel primo romanzo della serie, si presenta in commissariato insieme a un'altra donna svedese: "“Fettivamenti Catarella non

${ }^{26}$ Coprono il $17 \%$ di tutte le forme e il $5 \%$ di tutte le occorrenze. Vengono impiegati per marcare sintatticamente sia la narrazione che i dialoghi con le frasi relative libere introdotte da chi, come ecc.

${ }^{27}$ Coprono tutte insieme il $7 \%$ di tutte le forme e il $4 \%$ di tutte le occorrenze. 
aviva torto. 'Ntanto contrarimenti al modello svidisi 'sportato in tutto il munno non era biunna ma bruna bruna, la pelli bianca bianca, l'occhi cilestri cilestri, i capilli ricci ricci. Avuta, addisignata con un compasso che faciva curve perfette" (RP 98). Né cambiando scala letteraria vengono meno ripetitività ed effetto creato: "La matinata, sino dalla prim'alba, si era addimostrata volubili e capricciosa. Epperciò, per contagio, macari il comportamento di Montalbano, in quella matinata, sarebbi stato minimo minimo instabili" (LL 9). "La matinata sarebbi stata volubili e capricciosa. Epperciò, per contagio, macari il sò comportamento sarebbi stato minimo minimo instabili" (LL 256). A livello di paragrafo, incipit ed explicit del romanzo Una lama di luce sono pressoché identici. Come inizia il primo giorno della narrazione così inizia l'ultimo con un effetto di circolarità: laddove inizia, lì finisce il tempo del racconto. Anche a livello di syuzhet si possono notare gli stessi effetti in tanti testi. La maggior parte delle indagini del commissario Montalbano si risolve con il salta fosso o sfunnapedi, un trabocchetto impiegato per confermare in prove, indizi che diversamente resterebbero tali privando dell'effetto rassicurante dato dal crime storytelling camilleriano.

Ricorsività di cui sono colme le pagine camilleriane ritornano continuamente, in modo rassicurante e suadente, anche se inframezzate da omicidi e cadaveri. In Foucault Gilles Deleuze (1988) definisce l'attività critica come la ricerca dell'unicità e del raro, Camilleri invece sembra spingere alla ricerca del ripetuto. Un cuntastorie con narrazioni che sembrano tendere stilisticamente alla favola e alla fiaba, forme socialmente codificate dei primi stadi della ontogenesi della narrazione dai vecchi ai giovani. Una voce narrante in parte socialmente allineata alle caratteristiche di uno dei paesi al mondo con il più alto numero di anziani, che di conseguenza produce un maggior numero di "storytelling gerontologicamente connotati”. Una ridondanza narrativa scalare che nei decenni ha instaurato salde sinapsi linguistiche e narrative con i lettori, echeggiante, nella realtà, parte delle qualità dello storytelling socialmente più diffuso. 


\section{BIBLIOGRAFIA}

\section{LISTA DELLE ABBREVIAZIONI E FONTI BIBLIOGRAFICHE PRIMARIE}

(1994). La forma dell'acqua (65th ed.). Palermo: Sellerio.

FA

(1996a). Il cane di terracotta (62nd ed.). Palermo: Sellerio.

CANT

(1996b). Il ladro di merendine (66th ed.). Palermo: Sellerio.

LM

(1997). La voce del violino (50th ed.). Palermo: Sellerio.

VV

(1999a). Un mese con Montalbano (2014 ed.). Milano: Mondadori.

MM

La lettera anonima

la

L'arte della divinazione

La sigla

ad

Par condicio

si

Amore

pc

Una gigantessa dal sorriso gentile

a

Un diario del '43

gsg

L'odore del diavolo

d43

Il compagno di viaggio

od

Trappola per gatti

cv

Miracoli di Trieste

tg

Icaro

mt

L'avvertimento

Being here av

Il patto

bh

Quello che contò Aulio Gellio

Il vecchio ladro

La veggente

Guardie e ladri

vl

Tocco d'artista

$\mathbf{v}$

L'uomo che andava appresso ai funerali

gl

Una faccenda delicata

toca

uaaf

Lo Yack

fd

I due filosofi e il tempo

Cinquanta paia di scarpe chiodate

dft

Il topo assassinato

cpsc

Un angolo di paradiso

topa 
Capodanno

Lo scippatore sc

Movente a doppio taglio $\mathbf{m d t}$

(1999b). Gli arancini di Montalbano (21st ed.). Milano: Mondadori. $\quad$ AM

La prova generale

La pòvira Maria Castellino

pg

Il gatto e il cardellino

pmc

Sostiene Pessoa

gc

Un caso di omonimia

sop

Catarella risolve un caso

co

Il gioco delle tre carte

Pezzetti di spago assolutamente inutilizzabili

crc

Referendum popolare

gtc

Montalbano si rifiuta

psai

Amore e fratellanza

Sequestro di persona

af

Stiamo parlando di miliardi

Come fece Alice

sep

La revisione

spm

cfa

Una brava fimmina di casa

$\mathbf{r}$

"Salvo amato..." "Livia mia..."

bfe

La traduzione manzoniana

salm

Una mosca acchiappata al volo

tm

Gli arancini di Montalbano

mav

am

(2000). La gita a Tindari (45th ed.). Palermo: Sellerio.

GT

(2001). L'odore della notte (36th ed.). Palermo: Sellerio. ON

(2002). La paura di Montalbano (2014 ed.). Milano: Mondadori. $\quad$ PM

Giorno di febbre

gf

Ferito a morte

fm

Un cappello pieno di pioggia

cpp

Il quarto segreto

La paura di Montalbano $\quad$ pm

Meglio lo scuro ms

(2003). Il giro di boa (32nd ed.). Palermo: Sellerio. GB

(2004). La pazienza del ragno (19th ed.). Palermo: Sellerio. $\quad$ PR

(2005a). La prima indagine di Montalbano (14th ed.). Milano:

Mondadori.

PIM

Sette lunedì 
La prima indagine di Montalbano

Ritorno alle origini

(2005b). La luna di carta (16th ed.). Palermo: Sellerio.

pim

ro

(2006a). La vampa di agosto (17th ed.). Palermo: Sellerio.

LC

(2006b). Le ali della sfinge (9th ed.). Palermo: Sellerio.

VA

(2007). La pista di sabbia (3rd ed.). Palermo: Sellerio.

AS

(2008a). Il campo del vasaio (10th ed.). Palermo: Sellerio.

PS

(2008b). La finestra sul cortile. In A. Camilleri, Racconti di

CAV

Montalbano

fc

(pp. 477-504) (10th ed.). Milano: Mondadori.

(2008c). L'età del dubbio (3rd ed.). Palermo: Sellerio.

ED

(2009). La danza del gabbiano (7th ed.). Palermo: Sellerio.

DG

(2010a). La caccia al tesoro (3rd ed.). Palermo: Sellerio.

CACT

(2010b). Acqua in bocca. Roma: Minimum Fax.

AB

(2010c). Il sorriso di Angelica (2nd ed.). Palermo: Sellerio.

SA

(2011). Il gioco degli specchi (6th ed.). Palermo: Sellerio.

GIOCS

(2012a). Una lama di luce (3rd ed.). Palermo: Sellerio.

LL

(2012b). Una voce di notte. Palermo: Sellerio.

VN

(2012c). Una cena speciale. In Camilleri \& al., Capodanno in giallo (pp. 17-54) (2nd ed.). Palermo: Sellerio.

(2013a). Un covo di vipere (5th ed.). Palermo: Sellerio.

cs

COV

(2013b). Notte di Ferragosto. In Camilleri \& al., Ferragosto in giallo (pp. 13-50) (4th ed.). Palermo: Sellerio.

(2014a). La piramide di fango. Palermo: Sellerio.

PF

(2014b). Morte in mare aperto (4th ed.). Palermo: Sellerio.

La stanza numero 2

Doppia indagine

$M A$

Morte in mare aperto

sn2

di

Il biglietto rubato

La transazione

Come voleva la prassi

mma

Un'albicocca

br

t

cmp

Il ladro onesto

al

lo

(2015). La giostra degli scambi. Palermo: Sellerio.

GIOSS

(2016). L'altro capo del filo. Palermo: Sellerio.

ACF

(2017a). La rete di protezione. Palermo: Sellerio.

RP

(2017b). La calza della Befana. In Camilleri \& al., Un anno in giallo (pp. 9-46). Palermo: Sellerio.

cb 
(2018a). Il metodo Catalanotti. Palermo: Sellerio.

(2018b). Ventiquattr'ore di ritardo. In Camilleri \& al., Una giornata in giallo (pp. 9-41). Palermo: Sellerio.

(2019). Il cuoco dell'Alcyon. Palermo: Sellerio.

(2020). Riccardino. Seguito dalla prima stesura del 2005. Palermo: Sellerio.

$\mathbf{R I} / \mathbf{R}$

\section{FONTI BIBLIOGRAFICHE SECONDARIE}

Agnello Hornby, S. (2016). Come il ciclope del tempio di Zeus. Quaderni camilleriani. Il patto, 1, 29-31. Retrieved from https://www.camillerindex. it/quaderni-camilleriani/quaderni-camilleriani-1/.

Biundi, G. (1851). Vocabulario manuale completo siciliano-italiano. Palermo: Stamperia Carini. Retrieved from https://archive.org/details/vocabolariomanua00biun.

Bonfiglio, G. (2009). Siciliano-italiano. Piccolo vocabolario ad uso e consumo dei lettori di Camilleri e dei siciliani di mare. Roma: Fermento.

Buttitta, A. (intr.). (2004). Il caso Camilleri. Letteratura e storia. Palermo: Sellerio.

Camilleri, A. (1998). Il corso delle cose. Palermo: Sellerio.

Camilleri, A., \& De Mauro, T. (2014). La lingua batte dove il dente duole. Roma/Bari: Laterza.

Camilleri, A., \& Lodato, S. (2002). La linea della palma. Saverio Lodato fa raccontare Camilleri. Milano: Rizzoli.

Campailla, S. (Ed). (2012). Verga. Tutti i romanzi, le novelle e il teatro. Roma: Newton Compton editori.

Cerrato, M. (2012). L'alzata d'ingegno. Analisi sociolinguistica dei romanzi di Andrea Camilleri. Firenze: Franco Cesati.

Curcio, M. (Ed.). (2017). I fantasmi di Camilleri. Budapest: L'Harmattan.

Deleuze, G. (1988). Foucault. Minneapolis/London: University of Minnesota Press.

Demontis, S. (2001). I colori della letteratura. Un'indagine sul caso Camilleri. Milano: Rizzoli.

Guastella, F. (2015). Andrea Camilleri. Guida alla lettura. Acireale: Bonanno.

Marci, G. (Ed.). (2004). Lingua, storia, gioco e moralità di Andrea Camilleri. Cagliari: CUEC. 
Matt, L. (2020). Lingua e stile della narrativa camilleriana. Quaderni camilleriani. Parole, musica (e immagini), 12, 39-93. Retrieved from https:// www.camillerindex.it/quaderni-camilleriani/quaderni-camilleriani-12\%.

Milanesi, L. (2015). Dizionario etimologico della lingua siciliana. Milano: Mnamon.

Moravcsik, E. (1978). Reduplicative constructions. In J. H. Greenberg (Ed.), Universals of Human Language, vol. III (pp. 297-334). Stanford: Stanford University Press.

Mortillaro, V. (1853). Nuovo dizionario siciliano-italiano. Palermo: Pensante. Retrieved from https://archive.org/details/nuovodizionarios00mortuoft.

Mueller, M. (2014). Shakespeare His Contemporaries: collaborative curation and exploration of Early Modern drama in a digital environment. Digital Humanities Quarterly, 8(3). Retrieved from http://www.digitalhumanities.org $/ \mathrm{dhq} / \mathrm{vol} / 8 / 3 / 000183 / 000183 . \mathrm{html}$.

Nigro, S. S. (Ed.). (2004). Romanzi storici e civili. Milano: Mondadori.

Nigro, S. S. (Ed.). (2015). Gran Teatro Camilleri. Palermo: Sellerio.

Novelli, M. (Ed.). (2002). Storie di Montalbano. Milano: Mondadori.

Palumbo, O. (2005). L'incantesimo di Camilleri. Roma: Editori riuniti.

Piccitto, G. (1977-2002). Vocabolario siciliano. Catania/Palermo: CSFLS.

Rubino, C. (2005a). Reduplication: form, function, and distribution. In B. Hurch (Ed.), Studies on Reduplication (pp. 11-30), Berlin/New York: Mouton de Gruyter.

Rubino, C. (2005b). Reduplication. In M. Haspelmath, M. S. Dryer, D. Gil, \& B. Comrie (Eds.), The World Atlas of Language Structures (pp. 114-117). Oxford: Oxford University Press.

Santulli, F. (2010). Montalbano linguista. La riflessione metalinguistica nelle storie del commissario. Firenze: Arcipelago.

Scaglia, M. (2013). Il non siciliano di Andrea Camilleri. Roma: Viola.

Schwaiger, T. (2015). Reduplication. In P. O. Müller, I. Ohnheiser, S. Olsen, \& F. Rainer (Eds.), Word-formation. An International Handbook of the Languages of Europe, vol. I (pp. 467-484). Berlin/Boston: Mouton de Gruyter.

Sorgi, M. (2000). La testa ci fa dire. Dialogo con Andrea Camilleri. Palermo: Sellerio.

Stolz, T., Stroh, C., \& Urdze, A. (2011). Total reduplication: the areal linguistics of a potential universal. Berlin: Akademie Verlag.

Thornton, A. M. (2008). Italian Verb-Verb reduplicative Action Nouns. Lingue e linguaggio, 7(2), 209-232. 
Thornton, A. M. (2009). Italian verb reduplication between syntax and the lexicon. Italian Journal of Linguistics/Rivista di linguistica, 21(1), 235-261.

Trainito, M. (2008). Andrea Camilleri. Ritratto dello scrittore. Treviso: Anordest edizioni.

Valenti, I. (2014). Aspetti dell'inventività linguistica: Stefano D'Arrigo, Fosco Maraini, Andrea Camilleri. InVerbis, 4(1), 221-244.

Wierzbicka, A. (1986). Italian reduplication: Cross cultural pragmatics and illocutionary semantics. Linguistics, 24, 287-315.

Riassunto: Il contributo presenta i risultati di una ricerca sulla reduplicazione linguistica in vigatese, la lingua letteraria inventata dallo scrittore Andrea Camilleri. Un aspetto, questo, della narrazione camilleriana spesso rilevato dagli studiosi, ma che non ha ancora ricevuto uno studio dedicato. Viene riportata parte dei dati della ricerca condotta sul corpus dei 101 testi con protagonista il commissario Salvo Montalbano - composto da 29 romanzi e 72 racconti pubblicati tra il 1994 e il 2020, entro cui sono state registrate quasi 300 differenti forme reduplicative con più di 2.000 occorrenze totali. $\mathrm{Si}$ presentano i dati sulla reduplicazione espressiva con aggettivi: 64 forme impiegate dall'autore con 379 occorrenze totali - analizzate per differenti scale letterarie, al livello della frase e al livello del paragrafo. Vengono restituite le collocazioni entro il corpus cartaceo, le varianti ortografiche e se l'impiego avviene mimeticamente o diegeticamente. L'approccio metodologico è di natura mixed methods: a una tradizionale analisi di close reading segue una digital assisted text analysis (DATA) seguendo il concetto di scalable reading (Mueller, 2014). È stata operata una seconda analisi dei dati sull'asse diegetico/mimetico. I risultati ottenuti sono stati quindi impiegati per una comparazione data driven condotta sul corpus di tutte le opere narrative di Giovanni Verga.

I risultati mostrano che tre volte su quattro le reduplicazioni sono una ricorrenza diegetica. Nonostante il largo ricorso alla mimesi nei testi, la vera cifra dell'oralità dello storytelling camilleriano sembra risiedere altrove. La ricerca rientra in uno studio più ampio di tutti i testi narrativi sotto la generale ipotesi di ricerca che i corrispettivi formali della popolarità dello scrittore siano da individuare nella ripetitività criticata in origine, forse troppo frettolosamente.

Parole chiave: reduplicazione linguistica, letteratura, vigatese, siciliano, Camilleri 